\title{
Evaluation of Shallow Groundwater Function in Independent Watershed Based on ArcGIS
}

\author{
Jiamin JIAO ${ }^{\mathrm{a}}$, Guiguo $\mathrm{LI}^{\mathrm{b}}$, Xiaomin TENG ${ }^{\mathrm{a}}$, Yuxi $\mathrm{LI}^{\mathrm{c}}$ and Wanglin LI ${ }^{\mathrm{a}, 1}$ \\ a School of Water Conservancy and Environment, University of Jinan, Jinan, China \\ b Shandong Provincial Water Diversion Project Operation and Maintenance Center, \\ Jinan, China \\ c School of Hydrology and Water Resources, Hohai University, Nanjing, China
}

\begin{abstract}
According to the characteristics of physical geography and hydrogeology in Pingtan Island area, an independent regional groundwater evaluation system is established according to the four-layer model structure of target layer, criterion layer, attribute layer and index layer in the system. AHP (Analytic hierarchy process) is used to determine the weight value of each index in the study area, and a set of groundwater function evaluation system suitable for the island area is established. A groundwater function evaluation model with high systematic order degree is constructed and applied to the groundwater function evaluation of Pingtan Island. The results show that the groundwater in Luyangpu plain, Longwangtoupu plain, Lianjiupu plain and Qilipu plain can be developed and utilized well; The function of groundwater in the south of Aozhen and the foot of Junshan mountain is general, so we need to pay attention to the protection of ecological environment; The comprehensive function of groundwater in reclamation area, Huoshaogang salt area and Tianzhuyukou seawater intrusion area is weak, which is not suitable for exploitation and utilization. The research results are suitable for the evaluation of groundwater function in independent small areas of islands, and can provide technical support for the sustainable utilization of groundwater resources in similar islands.
\end{abstract}

Keywords. Groundwater evaluation system, ecological environment, sustainable utilization

\section{Introduction}

Groundwater function refers to the effect of the change of groundwater quality and quantity in time and space on the social and natural environment [1], mainly including three major functions of resources, ecology, and geological environment. Groundwater function evaluation is divided into target function evaluation and dominant function evaluation. The former shows the distribution characteristics of groundwater single function in the study area; The latter is to take the three functions of groundwater as the evaluation object, which can show the distribution characteristics of three functions in the study area. Therefore, groundwater function evaluation can provide basis for

${ }^{1}$ Corresponding Author, Wanglin LI, School of Water Conservancy and Environment, University of Jinan, Jinan, China; Email: cswlwe@163.com. 
groundwater exploitation and ensure the sustainable development and utilization of groundwater resources in Pingtan Island.

In terms of groundwater function evaluation, foreign studies mainly focus on groundwater resource evaluation and its sustainable development and utilization, but less on groundwater function evaluation [2-5]. Compared with foreign countries, the research of groundwater function evaluation in China started earlier, and the first round of national groundwater resource evaluation was carried out as early as the 1980s. In November 2004, the report issued by China Geological survey clearly put forward the basic principles, technical standards and groundwater evaluation model of groundwater function evaluation, and carried out key technical research [6]. In 2005, the "technical outline of groundwater function zoning" published by the Ministry of land and resources clearly described the research objects and standards of groundwater function evaluation, and applied them to the northwest of China to build the groundwater function evaluation system in Northwest China [7]. In 2006, Zhang Guanghui and others began to study the theory and method of regional groundwater function and sustainable utilization evaluation [8]. Since then, groundwater function investigation and evaluation work has been carried out successively in non karst areas such as North China Plain, Songliao plain and northwest inland basin [9-11] At present, domestic research on groundwater function evaluation in plain area is relatively mature, and a relatively complete evaluation index system has been formed [12]. However, for the island and other independent areas with special hydrogeological conditions and the actual situation of groundwater utilization, the groundwater function evaluation index is miscellaneous and the workload is large [13].

Therefore, Pingtan Island in Fujian Province is selected as the research area in this paper. According to the hydrogeological conditions of the island and the characteristics of groundwater development and utilization, the groundwater function evaluation method suitable for the island area is studied.

\section{Study Area}

\subsection{Topography}

Pingtan Island is located in the eastern sea area of Fujian Province, with an area of 295 $\mathrm{km}^{2}$, which is the largest island in Fujian Province. The topographic distribution is shown in figure 1 . The topography of Pingtan Island is mainly marine plain with gentle terrain. Junshan is the highest mountain in the county. There are three NNE trending hilly belts in the north of Pingtan Island, and the plains of dawopu, luyangpu, shangpanyang and lianjiupu are distributed among the hills; In the middle of Pingtan Island, there are longwangtoupu plain, qilipu plain and other coastal plains. In the south, there are many low hills, among which there are Yangpu, cangxiayang, dongkunyang and other small plains.

\subsection{Hydrometeorology}

Pingtan Island has the characteristics of subtropical marine monsoon climate. Although the annual rainfall is relatively large, the average annual rainfall is $1180.2 \mathrm{~mm}$, but the total evaporation (water surface and land surface) is $1880.0 \mathrm{~mm}$, which is far greater than the rainfall. Pingtan Island belongs to the region of water shortage and serious drought. 
In addition, Pingtan Island has a narrow territory, and its water system is not fully developed. Most of the rivers are seasonal rivers, with short streams and small flow. All the surface water flows into the sea, further aggravating the problem of surface water shortage.

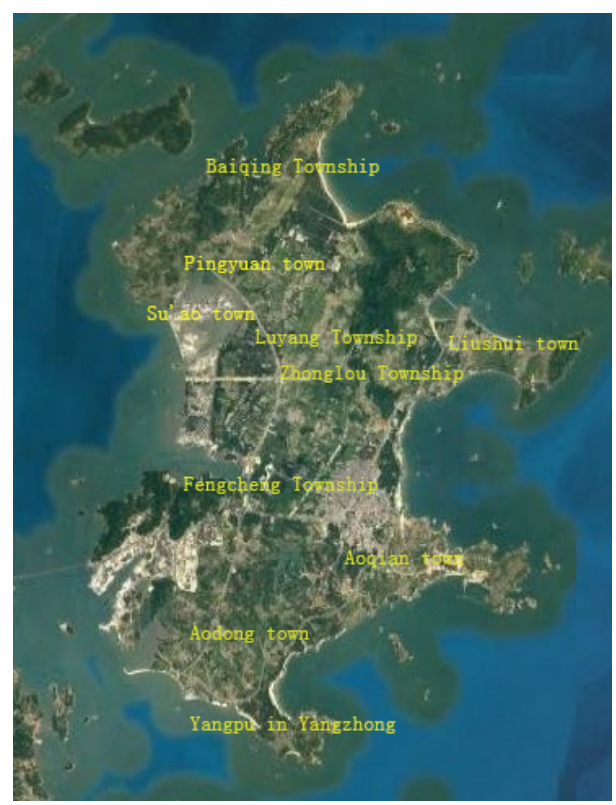

Figure 1. Topographic distribution of Pingtan Island.

\subsection{Geohydrologic Condition}

According to the water abundance law of groundwater in Pingtan Island, the water abundance of loose rock pore water in the study area can be divided into three areas: (1) Rich water quantity area: including Luyangpu plain, lianjiupu plain, Qilipu plain, Longwangtoupu plain and other places, mainly composed of marine sediments and Quaternary Holocene aeolian medium-fine sand, silt and medium-fine sand and gravel medium-coarse sand. The thickness of the aquifer is about 9 meters to 19 meters, and the depth of the water level is about half meters to one meter and five. (2) Middle water quantity area: mainly by Yangzhongyangpu, Cangxiayang, Budavopu, Yanxiapu, Dongkunyang, Zhifengyang and so on, Holocene aeolian, and fine sand, fine sand and medium fine sand and gravel containing coarse sand and so on several kinds of composition, survey shows that the aquifer usually thickness is about three meters to twelve meters, water level depth is generally one meter to two meters. (3) Poor water quantity area: mainly Suao, Aodong, Baiqing, and other hilly valleys, aquifer is mainly composed of aeolian, marine clay sand, silt and so on.

\subsection{Development and Utilization of Groundwater}

At present, the total land area of Pingtan Island is $371.9 \mathrm{~km}^{2}$, and the total amount of natural groundwater resources is $57.181 \mathrm{million}^{3} / \mathrm{a}$. due to the economic and technical conditions and traffic restrictions, it is difficult to plan and utilize the bedrock fissure 
water resources in some hilly areas. The economic and technical conditions for exploitation in Shapu plain are good, and the allowable exploitation amount can reach 36.5745 million $\mathrm{m}^{3} / \mathrm{a}$. If a large number of wells are reasonably planned and arranged, the amount of groundwater exploitation can be increased, which is of great significance to the "open source water for supply" of Pingtan County island.

\section{Method}

\subsection{Evaluation System Construction}

In order to study the groundwater function in Pingtan area from different layer, it is necessary to evaluate the groundwater function from four layer: index layer, system layer, attribute layer and function layer [13]. By means of expert consultation and experience analysis, the evaluation indexes suitable for the study area are selected from the index groups provided by the technical requirements for groundwater function evaluation and zoning. The selected evaluation indexes are combined to form the index layer (D). According to the rules, the attribute relationship of each index is clarified, and the attribute layer (C), the function criterion layer (B) is formed respectively, Finally, the groundwater function evaluation system suitable for island area is constructed.

According to the actual situation and existing data of groundwater utilization in Pingtan Island, a total of 13 indexes are selected to form the index layer of the whole evaluation system. The main aspects that need to be considered in the construction of attribute layer are as follows: (1) There is no large-scale stable water transfer in Pingtan Island, so the resource occupancy index of resource functional layer does not consider the share of external supply resources; There is a multiple relationship between the index of available resource occupancy and the index of recoverable resource modulus, so the index of available resource occupancy is abandoned. (2) There is only one medium-sized reservoir in the study area, and the reservoir area accounts for a small proportion of the total area of Pingtan Island. There is also a lack of detection data of water quality in the reservoir. Therefore, the water environment correlation in ecological function is eliminated. (3) The plain area of Pingtan Island is mainly sandy soil layer, but there is almost no land desertification phenomenon, so the evaluation index of land desertification and groundwater correlation degree is abandoned. (4) There is no geological disaster caused by subsidence in the study area, so the attribute index of geological environment stability in geological environment function is abandoned.

\subsection{Evaluation Index Empowerment}

Analytic hierarchy process (AHP) is a kind of analytical method which can quantify the qualitative analysis based on subjective judgment, and quantify the differences between judgment indexes. It can solve some complex problems with multi-objective, multi criteria and no structural characteristics. Compared with the scoring evaluation method, fuzzy comprehensive evaluation method and grey system evaluation method, the analytic hierarchy process has the advantages of systematicness, comprehensiveness, simplicity, and accuracy. Therefore, this paper uses the analytic hierarchy process to weight the groundwater function evaluation indexes. 


\subsubsection{Building Hierarchical Structure Model}

According to the analytic hierarchy process, the hierarchical structure of shallow groundwater function evaluation in Pingtan Island is constructed, as shown in figure 2.

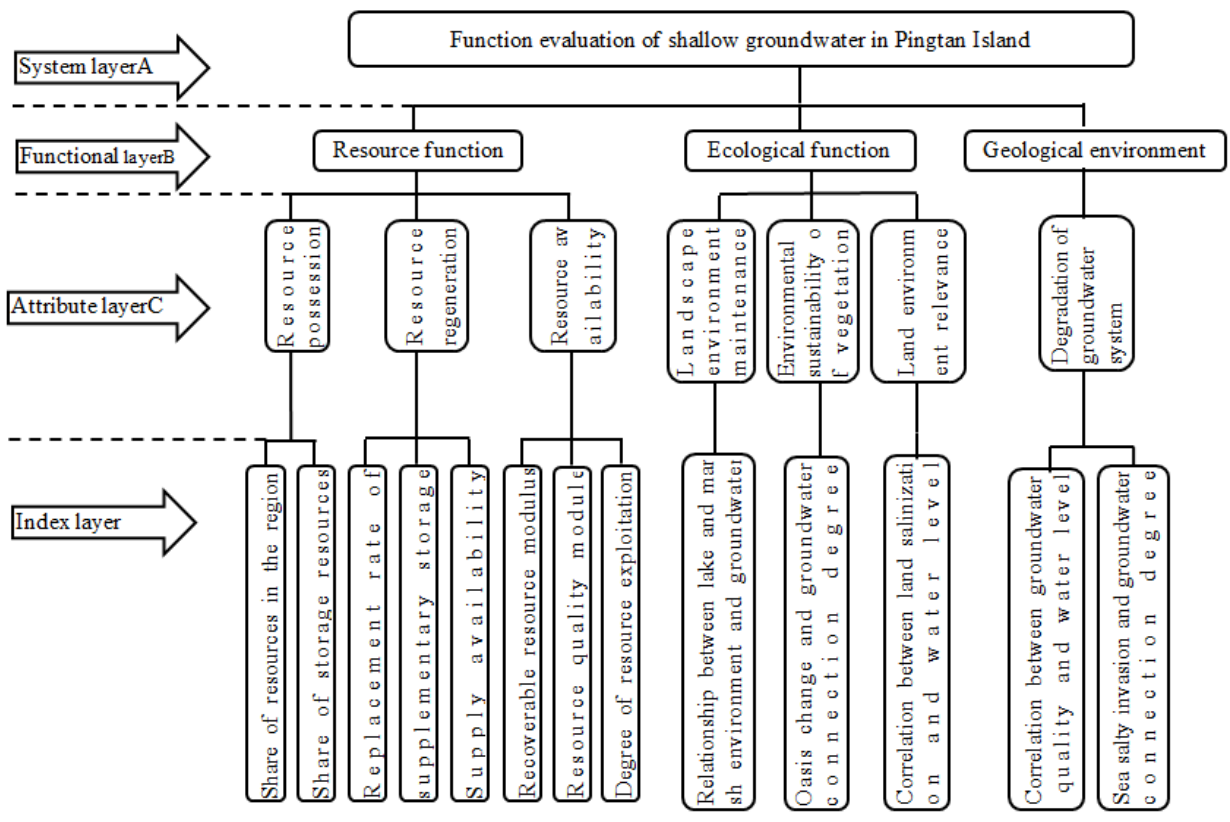

Figure 2. Hierarchical structure of shallow groundwater function evaluation in Pingtan Island.

\subsubsection{Construction of Judgment Matrix}

According to the constructed analytic hierarchy process (AHP) structure chart of Pingtan Island shallow groundwater function evaluation, combined with professional knowledge and expert experience, the importance of all indexes in the lower layer to the upper layer is assigned according to the 1-9 scale method, and the judgment matrix A (equation (1)) is constructed by pairwise comparison of $\mathrm{n}$ indexes in the lower layer dominated by the upper layer.

$$
\mathrm{A}=\left(a_{i j}\right)_{n \times n}
$$

$a_{i j}$ is the ratio of $x_{i}$ to $x_{j}$ for the previous layer. The main results of index layer, attribute layer and function layer are shown in tables 1-3 respectively.

Table 1. Index layer judgment matrix of resource occupancy indexes.

\begin{tabular}{lll}
\hline Resource possession & Supply resource share & Storage resource \\
\hline Supply resource share & 1 & $6 / 3$ \\
Storage resource & $3 / 6$ & 1 \\
\hline
\end{tabular}


Table 2. Judgment matrix of resource function attribute index layer.

\begin{tabular}{llll}
\hline Resource function & Resource possession & Resource regeneration & Resource availability \\
\hline Resource possession & 1 & $8 / 3$ & $8 / 5$ \\
Resource regeneration & $3 / 8$ & 1 & $3 / 5$ \\
Resource availability & $5 / 8$ & $5 / 3$ & 1 \\
\hline
\end{tabular}

Table 3. Judgment matrix of system target function index layer.

\begin{tabular}{llll}
\hline System target layer & Resource function & Ecological function & $\begin{array}{l}\text { Geological environment } \\
\text { function }\end{array}$ \\
\hline Resource function & 1 & $7 / 6$ & $7 / 4$ \\
Ecological function & $6 / 7$ & 1 & $6 / 4$ \\
Geological environment function & $4 / 6$ & $4 / 7$ & 1 \\
\hline
\end{tabular}

\subsubsection{Weight Calculation and Consistency Test}

After constructing the judgment matrix, calculate the weight vector of each judgment matrix (the weight value of each level index) according to equation (2). The vector form of relative weight is: $\omega=\left(\omega_{1}, \omega_{2}, \ldots \omega_{n}\right)^{\mathrm{T}}$.

$$
\omega_{i}=\frac{1}{n} \sum_{j}^{n} \frac{a_{i j}}{\sum_{k=1}^{n} a_{k j}}, \mathrm{i}=1,2,3, \ldots, \mathrm{n}
$$

After calculation, the weight values of each level in the groundwater function evaluation system of Pingtan Island are shown in table 4.

The consistency test of each judgment matrix is carried out according to the following steps.

(1) The largest eigenvalue of the matrix $\lambda_{\max }$ is calculated by equation (3):

$$
\lambda_{\max }=\frac{1}{n} \sum_{\mathrm{i}=1}^{\mathrm{n}} \frac{(\mathrm{A} \omega)_{\mathrm{i}}}{\omega_{\mathrm{i}}}
$$

$(\mathrm{A} \omega)_{\mathrm{i}}$ is the $I$ th component of $\mathrm{A} \omega$.

(2) Equation (4) is used for consistency test.

$$
\text { C. R. }=\frac{\text { C.I. }}{\text { R.I. }}
$$

Among them, the consistency index C.I. is calculated by the following formula:

$$
\text { C. I. }=\frac{\lambda_{\max }-n}{n-1}
$$

The consistency measure R.I. is found in table 5. 
Table 4. Weight calculation of each level in groundwater function evaluation system.

\begin{tabular}{|c|c|c|c|c|c|}
\hline Functional layer & Weig & tAttribute layer & Weig & tIndex layer & Weight \\
\hline & & & & Share of resources in the region & 0.67 \\
\hline & & Resource possession & 0.5 & Share of storage resources & 0.33 \\
\hline & & & & $\begin{array}{l}\text { Replacement rate of supplementary } \\
\text { storage }\end{array}$ & $\mathrm{y}_{0.18}$ \\
\hline Recource function & 0 & Resource regeneration & 0.19 & Supply availability & 0.35 \\
\hline Kesource iu & & & & $\begin{array}{l}\text { Balance ratio of supplementary } \\
\text { mining }\end{array}$ & $y_{0.47}$ \\
\hline & & & & Recoverable resource modulus & 0.44 \\
\hline & & Resource availability & 0.31 & Resource quality index & 0.31 \\
\hline & & & & Degree of resource exploitation & 0.25 \\
\hline & & $\begin{array}{l}\text { Landscape environm } \\
\text { maintenance }\end{array}$ & $\mathrm{t}_{0.17}$ & $\begin{array}{l}\text { Relationship between lake and marsh } \\
\text { environment and groundwater }\end{array}$ & \\
\hline Ecological function & 0.35 & $\begin{array}{l}\text { Environmental } \\
\text { sustainability of vegetati }\end{array}$ & 0.44 & $\begin{array}{l}\text { Oasis change and groundwater } \\
\text { connection degree }\end{array}$ & \\
\hline & & $\begin{array}{l}\text { Land environm } \\
\text { relevance }\end{array}$ & $t_{0.39}$ & $\begin{array}{l}\text { Correlation between land salinization } \\
\text { and water level }\end{array}$ & $n_{1}$ \\
\hline $\begin{array}{l}\text { Geological } \\
\text { environment function }\end{array}$ & 0.24 & $\begin{array}{l}\text { Degradation } \\
\text { groundwater system }\end{array}$ & $f_{1}$ & $\begin{array}{l}\text { Correlation between groundwater } \\
\text { quality and water level } \\
\text { Sea salty invasion and groundwater } \\
\text { connection degree }\end{array}$ & $\begin{array}{l}\mathrm{r}_{0.42} \\
\mathrm{r}_{0.58}\end{array}$ \\
\hline
\end{tabular}

Table 5. Average random consistency index.

\begin{tabular}{lllllll}
\hline $\mathbf{n}$ & $\mathbf{1}$ & $\mathbf{2}$ & $\mathbf{3}$ & $\mathbf{4}$ & $\mathbf{5}$ & $\mathbf{6}$ \\
\hline R.I. & 0 & 0 & 0.52 & 0.89 & 1.12 & 1.24 \\
$\mathrm{n}$ & 7 & 8 & 9 & 10 & 11 & 12 \\
R.I. & 1.36 & 1.41 & 1.46 & 1.49 & 1.52 & 1.54 \\
\hline
\end{tabular}

C. R. is the consistency ratio. If C.R. $<0.1$, the judgment matrix passes the consistency test; Otherwise, it failed. If not, the judgment matrix should be modified.

By calculation, the consistency test of each judgment matrix in Pingtan Island groundwater function evaluation system C.R. All of them are less than 0.1 , meeting the consistency test standard.

\subsection{Building a Comprehensive Evaluation Model}

\subsubsection{Model Selection}

When building a comprehensive evaluation and analysis model, two models, additive synthesis and multiplicative synthesis, are generally used. The additive synthesis model can complement each other for each index and reduce the influence of too low or too high index on the total score. The multiplicative synthesis of too low or too high value index will greatly affect the size of the total score. Therefore, this paper uses the weighted arithmetic average synthesis model of additive synthesis model to build the function evaluation model.

\subsubsection{Model Construction}

By combining the index weight $a_{i}$ with the evaluation parameters $x_{i}$ under the influence of a single index, the function evaluation index strength $\mathrm{R}$ of each sub-region can be obtained. 


$$
\mathrm{R}=\sum_{\mathrm{i}=1}^{\mathrm{n}} \mathrm{a}_{\mathrm{i}} \mathrm{x}_{\mathrm{i}}
$$

where $\mathrm{R}$ is the evaluation index. $\mathrm{a}_{\mathrm{i}}$ is the weight corresponding to the evaluation index. $\mathrm{x}_{\mathrm{i}}$ is the evaluation parameter.

\subsubsection{Grading of Functional Evaluation Results}

According to equation (6), the $\mathrm{R}$ value of each level index is calculated. In the hierarchy structure, the $\mathrm{R}$ value corresponding to each level is shown in table 6 . For different target functions, the development and utilization prospects of groundwater corresponding to $\mathrm{R}$ value are shown in table 7.

Table 6. Grading standards of groundwater function evaluation.

\begin{tabular}{llllll}
\hline Hierarchy Classification & Weak & Relatively weak & Commonly & Relatively strong & Strong \\
\hline System layer & & & & & \\
Functional layer & $0-0.2$ & $0.2-0.4$ & $0.4-0.6$ & $0.6-0.8$ & $0.8-1.0$ \\
Attribute layer & $0-0.17$ & $0.17-0.34$ & $0.34-0.67$ & $0.67-0.84$ & $0.84-1.0$ \\
\hline
\end{tabular}

Table 7. Classification and significance of groundwater target function evaluation results.

\begin{tabular}{lclll}
\hline Function name and code & Graded index & Functional status & Level & Utilization Prospect \\
\hline & $\mathrm{R}>0.84$ & Strong & I & Large scale mining \\
Resource function, & $0.67<\mathrm{R} \leq 0.84$ & Relatively strong & II & Moderate mining \\
$\mathrm{B}_{1}$ & $0.34<\mathrm{R} \leq 0.67$ & Commonly & III & Regulating mining \\
& $0.17<\mathrm{R} \leq 0.34$ & Relatively weak & IV & Not suitable for Mining \\
& $\leq 0.17$ & Weak & V & No mining \\
& $\mathrm{R}>0.84$ & Strong & I & Not available \\
& $0.67<\mathrm{R} \leq 0.84$ & Relatively strong & II & Not suitable for use \\
& $0.34<\mathrm{R} \leq 0.67$ & Commonly & III & Conservation and \\
Ecological functionB ${ }_{2}$ & $0.17<\mathrm{R} \leq 0.34$ & Relatively weak & IV & Modilization \\
& $\leq 0.17$ & Weak & V & Planning and Utilization \\
& $\mathrm{R}>0.84$ & Strong & I & No mining \\
& $0.67<\mathrm{R} \leq 0.84$ & Relatively strong & II & Not suitable for Mining \\
Geological environment & $0.34<\mathrm{R} \leq 0.67$ & Commonly & III & Regulating mining \\
function, $\mathrm{B}_{3}$ & $0.17<\mathrm{R} \leq 0.34$ & Relatively weak & IV & Moderate mining \\
& $\leq 0.17$ & Weak & V & Large scale mining \\
\hline
\end{tabular}

\section{Results}

\subsection{Target Function Evaluation Results of Shallow Groundwater}

In order to systematically characterize the distribution characteristics of each target function in the study area, based on the data calculation of each index unit of shallow groundwater in the study area, according to the established shallow groundwater function evaluation system and comprehensive evaluation model, combined with the corresponding weight of each index, the corresponding evaluation index of each attribute index is calculated, Then the evaluation index of each function index in each grid unit is calculated, and the target function evaluation of shallow groundwater in the study area is finally completed. The evaluation results are shown in figures 3-5. 


\subsection{Evaluation Results of Dominant Function of Shallow Groundwater}

The dominant function evaluation of groundwater is to take the resource function, ecological function and geological environment function of groundwater as the research object at the same time, and comprehensively reflect the distribution characteristics of the comprehensive function of shallow groundwater in the study area. Based on the target function evaluation of shallow groundwater resource function, ecological function and geological environment function in the study area, combined with the comprehensive function system and function index weight of shallow groundwater in the study area, the dominant function evaluation results of shallow groundwater are obtained, as shown in figure 6 .

\subsection{Discussion}

According to the evaluation results of shallow groundwater target and dominant function in Pingtan Island, it can be seen that:

- In the study area, the groundwater resources in Luyangpu Plain, Lianjiupu Plain, Longwangtoupu Plain and Qilipu Plains are relatively abundant, and the dominant part is the resource function, and the corresponding ecological and geological environment functions can be weakened.

- The ecological function of the south of Zhongsuao Town, the south of Aoqian town and Aodong town and the natural ecological protection area of 36 foot lake are relatively strong, while the resource function and geological environment function are relatively weak.

- The reclamation area, Huoshaogang salt field area and Zhuyukou seawater intrusion area have strong geological environment function, weak resource function and weak ecological function.

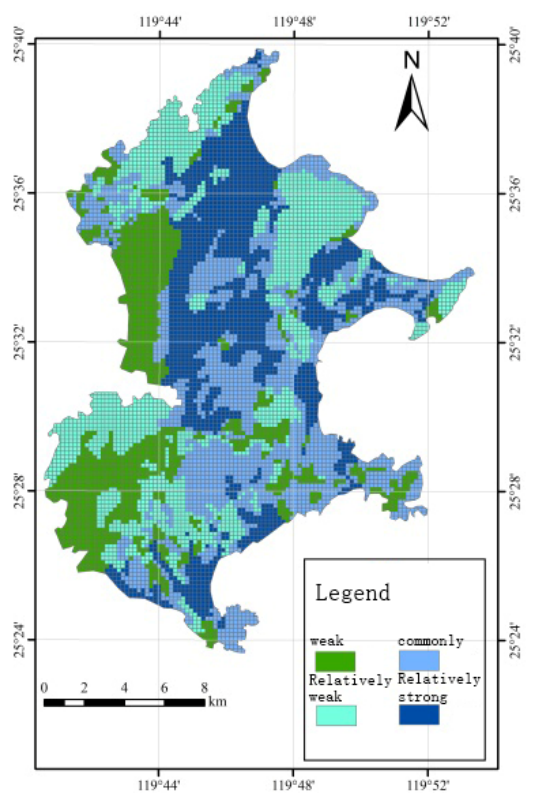

Figure 3. Evaluation result of resource function.

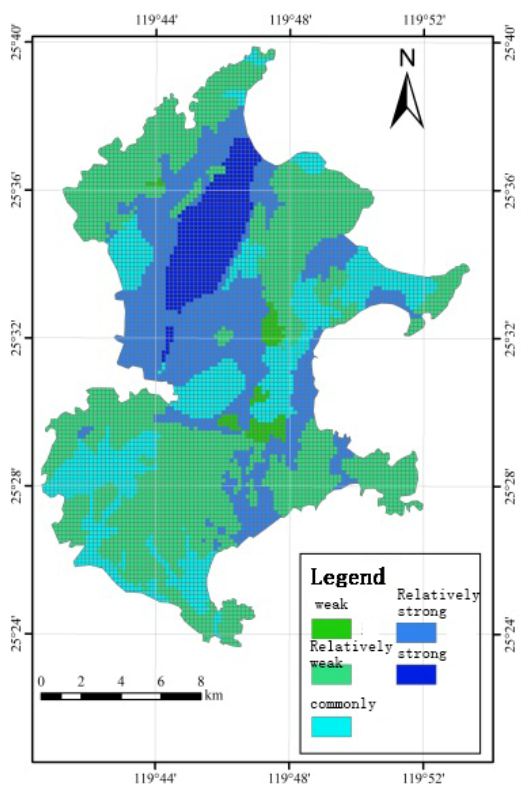

Figure 4. Evaluation result of ecological function. 


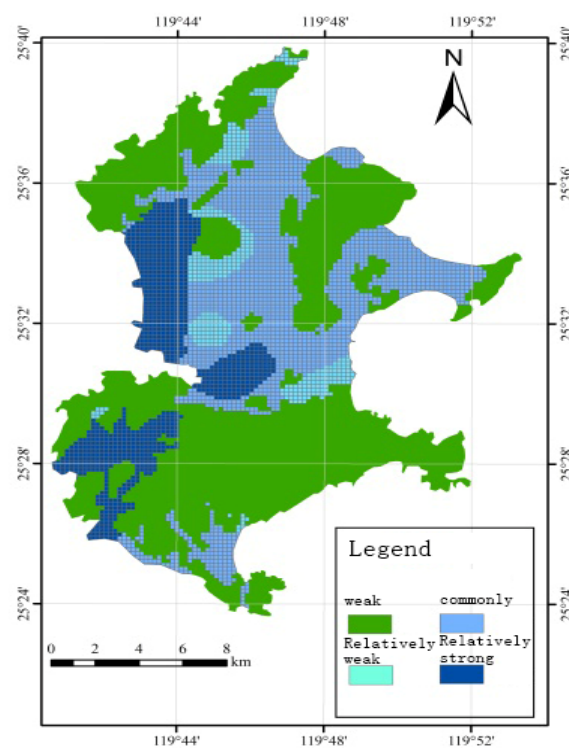

Figure 5. Evaluation results of geological environment function.

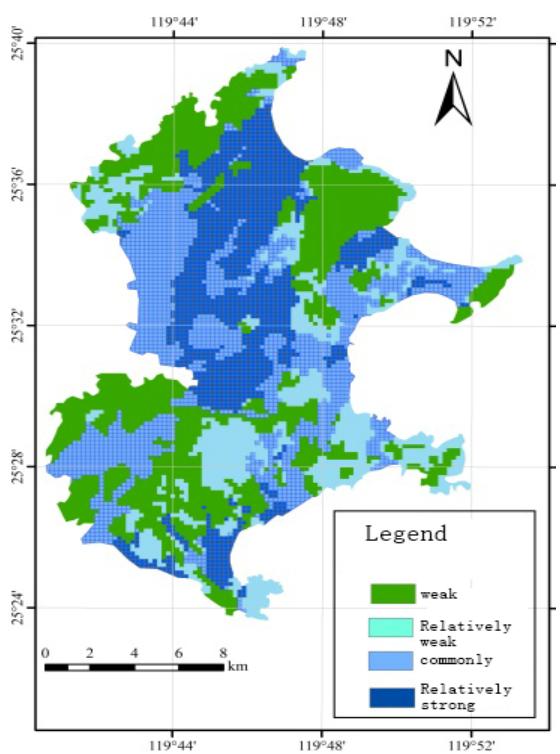

Figure 6. Comprehensive results of leading functions.

\section{Conclusion}

Based on the special topography, meteorology, hydrology, hydrogeology, and the actual situation of groundwater utilization in Pingtan Island, 13 dominant indexes, such as the share of recharge resources and the share of storage resources, are selected from 34 indicators of groundwater function evaluation system in general areas, A groundwater function evaluation index system suitable for small island area is established.

The evaluation results show that the study area has a large area with strong resource function, which is worthy of attention in the future development and utilization of groundwater resources. Weak ecological function is the most widely distributed, so it is necessary to strengthen the ecological environment protection and governance measures. The general geological function areas are widely distributed in the study area, and the rest of the functional status areas are distributed. Large areas of weak geological function areas need to pay attention to the protection of geological environment. The exploitation of groundwater is also easy to lead to problems of geological environment, so it is necessary to limit the exploitation, and scientific geological environment protection measures can be taken.

\section{Acknowledgements}

This work was financially supported by the Shandong Provincial Natural Science Foundation, China (ZR2019MEE106). 


\section{References}

[1] Yuan Y and Tang Y 2020 Groundwater function evaluation of Turpan City Journal of China Academy of Water Resources and Hydropower Sciences 18 (04) 284-294.

[2] Catarina and Roseta-Palma 2003 Joint quantity management of groundwater Journal of Environmental and Resource Economics (26) 89-106.

[3] Sophocleous M 2000 From safe yield to sustainable development of water resources-the Kansas experience Journal of Hydrology 235 (1-2) 27-43.

[4] Frans R P and Woolley K D R 2005 Applicability and methodology of determining sustainable yield in groundwater systems Journal of Hydrogeology (13) 295-312.

[5] Fan W 2007 Groundwater function evaluation in plain area of Jilin Province Conference of Jilin University.

[6] Zhang G Shen J and Nie Z 2006 Evaluation theory and method of regional groundwater function and sustainable utilization. Hydrogeology and Engineering Geology 33 (4) 62-66 71.

[7] Li F and Peng H and Li M 2013 Study on groundwater function evaluation and regionalization in Tianjin Journal of safety and environment 13 (03) 111-115.

[8] Nie Z, Zhang G, Shen J, Yan M and Wang J 2012 Functional characteristics and sustainable utilization of groundwater in Northwest Inland Basin Journal of Resources and Environment in Arid Area 26 (01) 63-66.

[9] Xiao P, Yu W, Hu G and Zhao X 2017 Groundwater function evaluation and regionalization in Jianghan Dongting Plain Journal of Renmin Changjiang River 48 (01) 6-11+19.

[10] Zhang L, Liu X and Yan Y 2010 Screening of groundwater function evaluation indexes in southern China: A case study of Guangdong Province Journal of Water Resources Protection 26 (04) 26-29+94.

[11] Nie Z, Zhang G, Shen J, Hao M and Wang J 2007 Development and application of visualization platform for groundwater function evaluation Journal of Earth (06) 579-584.

[12] Yang J 2016 Study on Groundwater Function Evaluation and Regulation Scheme in Tianjin (North China University of Water Resources and Hydropower).

[13] Sun C and Li X 2013 Groundwater function evaluation of Xialiaohe Plain based on ArcGIS Journal of Geoscience 33 (02) 174-180. 